\title{
Tau and atrophy: domain-specific relationships with cognition
}

\author{
Leonardino A. Digma'1, John R. Madsen', Emilie T. Reas ${ }^{1}$, Anders M. Dale ${ }^{1,2,3,4}$, James B. Brewer ${ }^{1,2}$, \\ Sarah J. Banks ${ }^{1,4^{*}}$ and for the Alzheimer's Disease Neuroimaging Initiative
}

\begin{abstract}
Background: Late-onset Alzheimer's disease (AD) is characterized by primary memory impairment, which then progresses towards severe deficits across cognitive domains. Here, we report how performance in cognitive domains relates to patterns of tau deposition and cortical thickness.

Methods: We analyzed data from 131 amyloid- $\beta$ positive participants (55 cognitively normal, 46 mild cognitive impairment, $30 \mathrm{AD}$ ) of the Alzheimer's Disease Neuroimaging Initiative who underwent magnetic resonance imaging (MRI), flortaucipir (FTP) positron emission tomography, and neuropsychological testing. Surface-based vertex-wise and region-of-interest analyses were conducted between FTP and cognitive test scores, and between cortical thickness and cognitive test scores.
\end{abstract}

Results: FTP and thickness were differentially related to cognitive performance in several domains. FTP-cognition associations were more widespread than thickness-cognition associations. Further, FTP-cognition patterns reflected cortical systems that underlie different aspects of cognition.

Conclusions: Our findings indicate that AD-related decline in domain-specific cognitive performance reflects underlying progression of tau and atrophy into associated brain circuits. They also suggest that tau-PET may have better sensitivity to this decline than MRI-derived measures of cortical thickness.

Keywords: Tau, Atrophy, Thickness, Positron emission tomography, Magnetic resonance imaging, Neuropsychology, Cognition, Surface-based analysis

\section{Background}

Alzheimer's disease (AD) typically presents clinically as an amnestic disorder with subsequent progression to other cognitive domains such as visuospatial abilities, language, and executive function [1]. The domains of cognition rely on distinct cortical systems, where distributed and specialized brain circuitry serves associated cognitive functions [2]. Degeneration and dysfunction of these large-scale cortical systems parallel the decline of specific cognitive domains observed in dementia [3].

Pathological events in AD are considered to cause the clinical manifestations through sequential and synergistic build-up of pathological proteins, amyloid- $\beta(\mathrm{A} \beta)$ and tau,

\footnotetext{
* Correspondence: sbanks@ucsd.edu

1 Department of Neurosciences, University of California, San Diego, 9500

Gilman Drive, La Jolla, CA 92093, USA

${ }^{4}$ Department of Psychiatry, University of California, San Diego, 9500 Gilman

Drive, La Jolla, CA 92093, USA

Full list of author information is available at the end of the article
}

in the brain and neurodegeneration. Although there is some evidence that elevated levels of $A \beta$ are associated with future cognitive decline [4], regional $A \beta$ is generally a poor correlate of cross-sectional cognition $[5,6]$. Tau and neurodegeneration, on the other hand, have been shown to be closely linked to deficits in cognition $[7,8]$. While the associations with tau have been determined through studies of post-mortem brain tissue [9], we are now able to study them in vivo using PET ligands $[10,11]$.

Autopsy data indicate that the spread of tau pathology follows a progressive sequence [12] consistent with the observed clinical course in AD: tau starts in the medial temporal lobe regions that are responsible for learning and memory, then into the rest of the cortex to affect the cortical areas that serve other cognitive domains. Initial studies using tau-binding radiotracers have recapitulated the Braak staging of tau that has been described in autopsy studies $[13,14]$, establishing the promise of these radiotracers for

(c) The Author(s). 2019 Open Access This article is distributed under the terms of the Creative Commons Attribution 4.0 International License (http://creativecommons.org/licenses/by/4.0/), which permits unrestricted use, distribution, and 
assessing, in vivo, how tau pathology relates to domain-specific cognitive decline.

Assessment of neurodegeneration in vivo has been available with high-resolution magnetic resonance imaging (MRI) measures of cortical thickness to estimate structural atrophy and with fluorodeoxyglucose (FDG)-PET to evaluate regional hypometabolism. Studies using these modalities have consistently shown that $\mathrm{AD}$ is characterized by degeneration in temporal and parietal cortices [15-18] in a pattern similar to tau pathology. Furthermore, in vivo studies of neurodegeneration, like post-mortem studies of tau, have revealed close associations between the distribution of neurodegeneration and cognitive deficits found in patients with $\mathrm{AD}[3,19-23]$.

Taken together, studies of tau and neurodegeneration suggest that the two are closely related and that both contribute to variability in cognitive performance. The relationship between neurodegeneration and cognition across different domains in vivo has been explored extensively, but the link between tau and cognition across domains in vivo remains to be fully characterized. Studies of tau-PET in early-onset atypical variants of $\mathrm{AD}$, such as primary progressive aphasia or posterior cortical atrophy, have shown that tau accumulation in cortical systems that serve specific cognitive domains is associated with impairment in those respective domains $[24,25]$. Interestingly, the aggregation of tau within functional cortical systems [26] and the association of tau with cognitive loss in different domains [27] has recently been demonstrated to similarly occur in lateonset, amnestic AD. In this study, our interest was to further examine how in vivo measures of tau and neurodegeneration associate with different domains of cognition in the common, amnestic AD phenotype.

To this end, we used multi-modal imaging and neuropsychological battery data available in the Alzheimer's Disease Neuroimaging Initiative (ADNI) to investigate the relationship between cross-sectional measures of tau, cortical thickness, and different aspects of cognition. We hypothesized that tau and cortical thickness would both correlate with cognition in a domain-specific manner, with tau demonstrating stronger associations over a wider area of the domain-relevant cortex. We tested these hypotheses by performing surface-based vertexwise analysis and region-of-interest (ROI) analysis in a group of $A \beta$-positive older adults with a diagnosis of cognitively normal, mild cognitive impairment, or AD.

\section{Methods}

\section{Study participants}

Our sample consisted of subjects from phase 2 and phase 3 of the ADNI. The ADNI was launched in 2003 as a public-private partnership, led by Principal Investigator Michael W. Weiner, MD, with the primary goal of testing whether serial imaging, biomarker, and clinical and neuropsychological assessments can be combined to measure the progression from $\mathrm{MCI}$ to $\mathrm{AD}$. For up-to-date information, see www.adni-info.org. Inclusion and clinical diagnosis criteria for ADNI have been described previously [28, 29]. We included subjects from ADNI who received MRI, tau-PET (flortaucipir-PET, FTP-PET), and $\mathrm{A} \beta$-PET (florbetapir- or florbetaben-PET) and underwent full neuropsychological testing, and had these data available in the ADNI data repository in August 2018, when our study began. Only subjects that were $A \beta$ positive, as determined by A $\beta$-PET and a standard threshold $[30,31]$ were included for statistical analysis to focus our study on participants who were on the AD pathophysiological continuum [32]. The A $\beta$-PET scan that was acquired closest in time to the tau-PET (time between A $\beta$-PET and tauPET: mean $=1.12$ years, $\mathrm{SD}=1.57$ years) scan was used to determine $A \beta$ positivity.

\section{MRI acquisition and processing}

An overview of the image processing steps used in our study is outlined in Fig. 1. Briefly, raw T1 MR images were downloaded in DICOM format from the ADNI data portal. Spatial distortions in MR images resulting from gradient nonlinearities were corrected using scanner-specific parameters [33]. Corrections for nonuniformity in signal intensity were then applied [34]. FreeSurfer (version 6.0) was used to automatically segment and parcellate each structural MRI, reconstruct the cortical surface, and measure thickness at each vertex on the cortical mantle $[35,36]$.

We also defined each subject's inferior cerebellar gray matter, which was used as the reference region in PET analyses (see the "PET acquisition and processing" section). The MRI was segmented into tissue probability maps with SPM12 (www.fil.ion.ucl.ac.uk/spm). Then, the spatially unbiased atlas template of the cerebellum and brainstem toolbox (SUIT, http://www.diedrichsenlab. org/imaging/suit.htm) was used to reverse normalize the SUIT template to the participants' native space MRI.

\section{PET acquisition and processing}

FTP-PET was conducted to estimate the extent of tau pathology. FTP-PET images were downloaded from the ADNI portal in the most preprocessed form: realigned to the first frame, averaged across frames, voxel sizes standardized, and resolution made uniform across sites [37]. These preprocessed images were coregistered to the subject's temporally closest MRI. These co-registered FTP-PET images were then converted into standard uptake value ratio (SUVr) images by normalizing the images to mean FTP uptake in the inferior cerebellar gray matter [38]. The individual SUVr volumes were then projected onto each individual's cortical surface model by sampling from points half-way between the white and pial surfaces. 


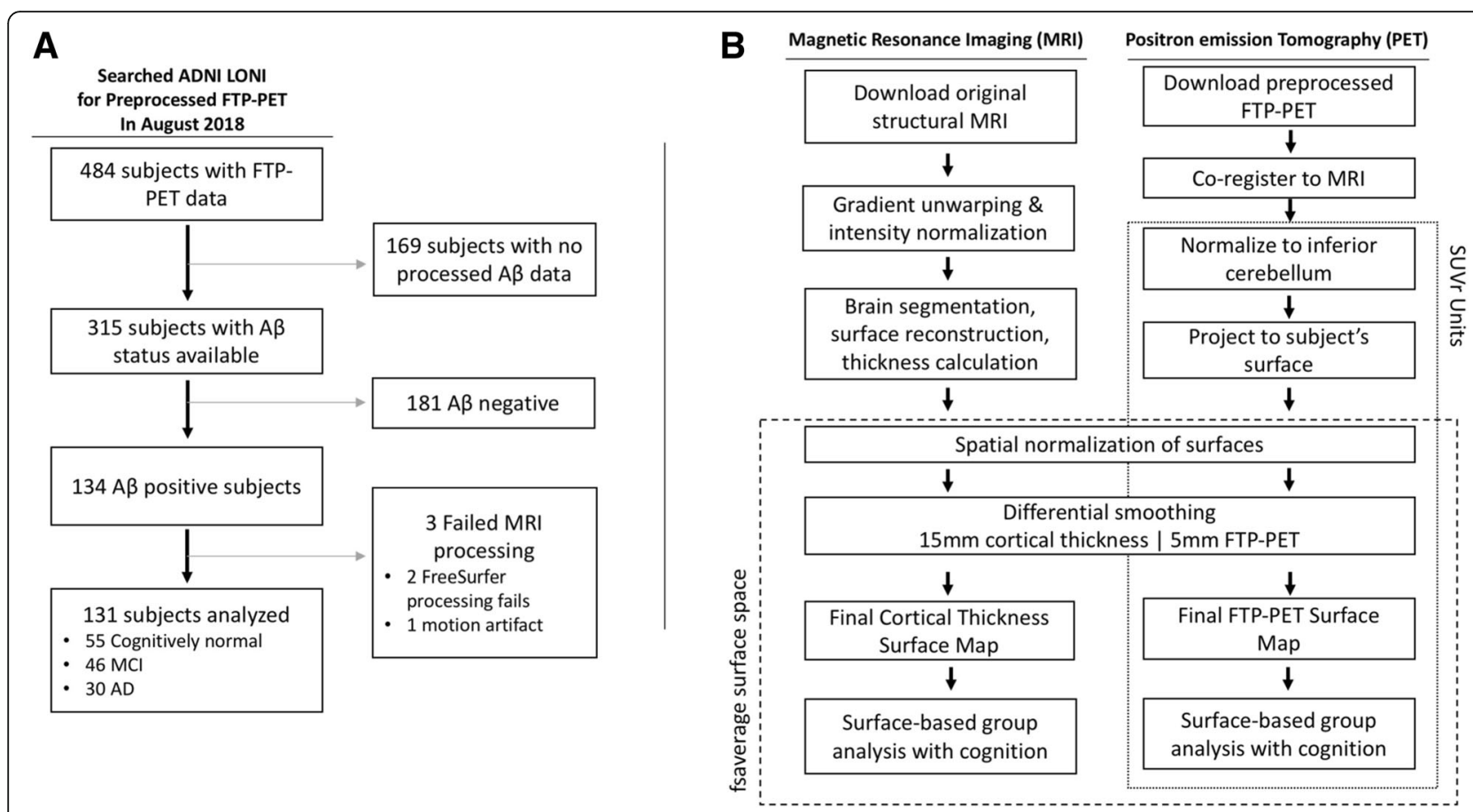

Fig. 1 Flow diagrams for subject inclusion and processing of neuroimaging data in our study. a The steps used for assembling the study sample. b The steps used for image processing for surface-based vertex-wise analysis. Abbreviations: SUVr: standard uptake value ratio; FTP: flortaucipir; mm: millimeters

\section{Processing for surface-based analyses}

Prior to conducting vertex-wise analyses (see the "Statistical analysis" section), the individual thickness maps and FTP maps were brought into a common space (fsaverage space) using spherical-based registration methods [39]. Furthermore, because cortical thickness and FTP datasets differed in spatial resolution in the surface maps, differential smoothing was applied to the thickness and FTP surface maps to obtain similar spatial resolutions along the cortical mantle prior to surface-based analysis. Kernels of $15 \mathrm{~mm}$ and $5 \mathrm{~mm}$ were used for the thickness and FTP maps, respectively.

\section{Processing for region-of-interest analyses}

In addition to surface-based vertex-wise analyses, we also performed ROI analyses using partial volume corrected (PVC) regional FTP data. The Geometric Transfer Matrix [40] approach for PVC was applied, in combination with FreeSurfer-parcellated ROIs from the Desikan-Killiany atlas [41] and SPM12 tissue segmentations as described previously [38]. For regional cortical thickness, we used the average thickness of each Desikan-Killiany ROI as outputted by FreeSurfer.

\section{Neuropsychological assessment}

Participants underwent neuropsychological testing to assess performance across cognitive domains. We used scores from their exam that was closest in time to their
FTP-PET date (time between neuropsychology visit and FTP-PET: mean $=0.60$ years, $S D=0.78$ years). We used various tests scores to represent the domains in question: the Rey Auditory Verbal Learning Test (RAVLT) and Logical Memory (LM) tests were used to assess learning and memory. We analyzed the RAVLT sum of all correctly learned words across trials 1 through 5 (RAVLT learning), RAVLT delayed recall, LM immediate recall, and LM delayed recall. To assess verbal fluency and naming abilities, we examined scores from the category fluency: animals and the Multilingual Naming Test (MINT). Lastly, to assess constructional abilities, processing speed, and executive function, we analyzed clock drawing score, clock copying score, and scores from parts A and B of the Trail Making Test (TMT), respectively. For all statistical analyses that involved TMT Part A or TMT Part B, scores were multiplied by - 1 so that lower scores indicated worse cognition.

\section{Statistical analysis \\ Surface-based analysis}

We performed surface-based general linear model analyses as implemented in FreeSurfer to explore the relationships between tau and cognition, and between cortical thickness and cognition. We used surfaced-based analysis because surface-based registration offers a more accurate alignment of cerebral cortex across subjects than volumebased registration [42] and because $2 \mathrm{D}$ smoothing across 
the surface helps prevent smoothing across different tissue types in the PET data [43]. Vertex-wise analyses were performed between FTP-PET and scores on each cognitive test, and between cortical thickness and scores on each cognitive test, with regressors for age, sex, education, and time-delay between scan and cognitive test administration. Model contrasts tested the hypothesis that more tau or thinner cortex (in separate models) was associated with worse cognitive performance. A Monte Carlo simulation method [44] was implemented for cluster exclusion and multiple comparisons correction, using a cluster-forming threshold of $-\log (P$ value $)>4$ or $P$ value $<0.0001$, and a clusterwise threshold $P$ value $<0.05$. All statistical surface maps are displayed at a threshold of $-\log (P$ value $)>4$ or $P$ value $<0.0001$ unless otherwise specified. We also performed diagnosis-stratified surface-based analyses, within $\mathrm{CN}, \mathrm{MCI}$, and $\mathrm{AD}$ subjects.

\section{Domain-specificity analysis}

Visual inspection of the FTP-cognition association maps suggested that there may exist cognitive domain-specific FTP retention patterns. To this end, we performed post hoc pairwise Meng's tests [45] between the FTP-cognition partial correlations maps (vertex-wise $P$ value $<0.005$ ) [46]. Meng's test compares two dependent correlations and allows us to determine whether FTP is more related to one cognitive test versus another at each point on the cortical surface. Meng's test was performed pairwise for the statistical maps from FTP vertex analyses with RAVLT learning, RAVLT delayed recall, category fluency, and TMT part A. These 4 tests were chosen because they had qualitatively similar topographical associations with tau as for LM immediate recall, LM delayed recall, MINT, and clock drawing, respectively. To limit the number of vertices examined, Meng's test was only performed in vertices that survived cluster correction in either of the analyses being compared.

The domain-specificity analysis was also performed for the partial correlation maps from the surfacebased thickness-cognition analyses. The same subset of tests-RAVLT learning, RAVLT delayed recall, category fluency, and TMT part A-were used for the domain-specificity thickness analyses. These results are reported in Additional file 1.

\section{Region-of-interest analyses}

To re-capitulate our vertex-wise analysis results at the ROI level, we performed ROI correlational analyses. We computed partial correlations between regional PVCFTP and cognitive test score, after each variable was regressed onto age, sex, education, and time-delay between the date of scan and date of cognitive test administration. This correlational analysis was done for each
ROI and cognitive test pair. This analysis was repeated for regional thickness, instead of regional PVC-FTP.

To explore the question of whether regional tau was associated with cognition, independently of regional thickness, we performed the following ROI analysis. We again computed partial correlations between regional PVC-FTP and cognitive test score. However, in this case, we regressed each variable onto regional thickness, in addition to age, sex, education, and time-delay between scan and cognitive test. This allowed the examination of associations between regional PVC-FTP and cognition, independent of regional thickness. Results from our ROI analyses are displayed in correlation matrices in Fig. 5.

In these analyses, we excluded FreeSurfer-defined regions that correspond to Braak stage 6 [13] as they likely exhibit little variability in FTP signal across the group. We applied a threshold of $P$ value $<9.9 \mathrm{e}-5$ to each correlation matrix, which corresponds to a family-wise error (FWE) corrected $P$ value of 0.05 for each matrix. Lastly, in the PVC methods we used, medial and lateral orbitofrontal were combined into a single ROI, pars orbitalis, opercularis, and triangularis were combined into a single ROI, and rostral and caudal middle frontal were combined into a single ROI.

\section{Results \\ Subject characteristics}

A total of 134 ADNI participants met study criteria $(A \beta$ positive, received tau-PET imaging, received MRI, and underwent the neuropsychology battery). Three subjects were excluded as their structural MRI did not successfully process through FreeSurfer or their MRI had substantial motion artifact, leaving us with 131 participants to examine. Of these participants, 55 had a diagnosis of cognitively normal, 46 with mild cognitive impairment, and 30 with AD. The average participant age was $77.8( \pm 7.3)$ years and 65 of the participants were female. Participant demographics and a summary of cognitive test scores are shown in Table 1.

\section{Associations between tau and cognitive function Learning and Memory}

In general, worse scores on the RAVLT and LM were associated with more FTP binding (Fig. 2a). Visual inspection of the statistical maps revealed that lower cognitive scores were associated with FTP in medial, inferior, lateral temporal cortex and also in medial and lateral parietal cortex. Notably, there were stronger associations in frontal regions for RAVLT learning and LM immediate recall than for RAVLT delayed recall and LM delayed recall, respectively.

\section{Fluency and naming}

Lower performance on category fluency and MINT was also associated with greater FTP, as shown in Fig. 2b. 
Table 1 Subject demographics and cognitive test scores by diagnostic group

\begin{tabular}{llllll}
\hline Variable & CN & MCl & AD & All subjects & Domains tested \\
\hline N (\%female) & $55(56.3 \%)$ & $46(39.1 \%)$ & $30(53.3 \%)$ & $131(49.6 \%)$ & \\
Age & $77.3(6.3)$ & $77.5(7.6)$ & $79.0(8.5)$ & $77.8(7.3)$ & \\
RAVLT learning & $45.7(10.9)$ & $31.9(9.0)$ & $25.6(9.9)$ & $36.3(13.1)$ & Learning \\
RAVLT delayed recall & $7.7(4.7)$ & $2.8(3.5)$ & $1.5(3.0)$ & $4.6(4.8)$ & Memory \\
LM immediate recall & $15.0(3.2)$ & $9.8(4.9)$ & $6.0(5.25)$ & $11.1(5.7)$ & Memory \\
LM delayed recall & $14.0(3.7)$ & $7.5(5.2)$ & $2.8(4.6)$ & $9.1(6.3)$ & Memory \\
Category fluency_animals & $22.0(5.1)$ & $16.5(5.2)$ & $12.5(4.5)$ & $17.9(6.3)$ & Language \\
MINT* & $30.2(2.4)$ & $28.6(3.9)$ & $26.4(5.1)$ & $28.9(3.8)$ & Language \\
Clock drawing & $4.8(0.5)$ & $4.2(0.9)$ & $3.8(1.6)$ & $4.4(1.1)$ & Visuospatial \\
Clock copying & $4.7(0.8)$ & $4.7(0.5)$ & $4.3(1.1)$ & $4.6(0.8)$ & Visuospatial \\
Trail Making Test A & $31.9(7.7)$ & $41.3(19.4)$ & $60.3(36.2)$ & $41.7(24.0)$ & Executive function and processing speed \\
Trail Making Test B $^{\dagger}$ & $81.6(40.2)$ & $128.5(82.2)$ & $202.2(99.3)$ & $125.7(86.6)$ & Executive function and processing speed \\
\hline
\end{tabular}

Values are mean \pm SD unless otherwise noted

Abbreviations: $N$ number of participants, SD standard deviation, RAVLT Rey Auditory Verbal Learning Test, LM logical memory, MINT Multilingual Naming Test, CN cognitively normal, $M C I$ Mild Cognitive Impairment, $A D$ Alzheimer's disease

*Since MINT was added to the ADNI neuropsychology battery only recently, MINT scores were missing for 26 of our study participants

${ }^{\dagger}$ In the Trail Making Test, a higher score denotes worse performance

Significant correlations were present in the left frontal cortex and temporal, parietal, and lateral occipital cortex and were mostly left lateralized (Additional file 1: Figure S3).

\section{Visuospatial, executive function, and processing speed}

Compared to learning, memory, fluency, and naming tests, the clock copying and TMT performance had greater associations with FTP in posterior regions, including more superior parietal cortex (Fig. 2c). In addition, clock drawing, clock copying, and TMT part A also involved the medial temporal cortex to a lesser extent (Fig. 2c) than tests of learning, memory, and language. Compared to TMT part A, the association between FTP and TMT part B had more inferior and medial temporal lobe involvement.

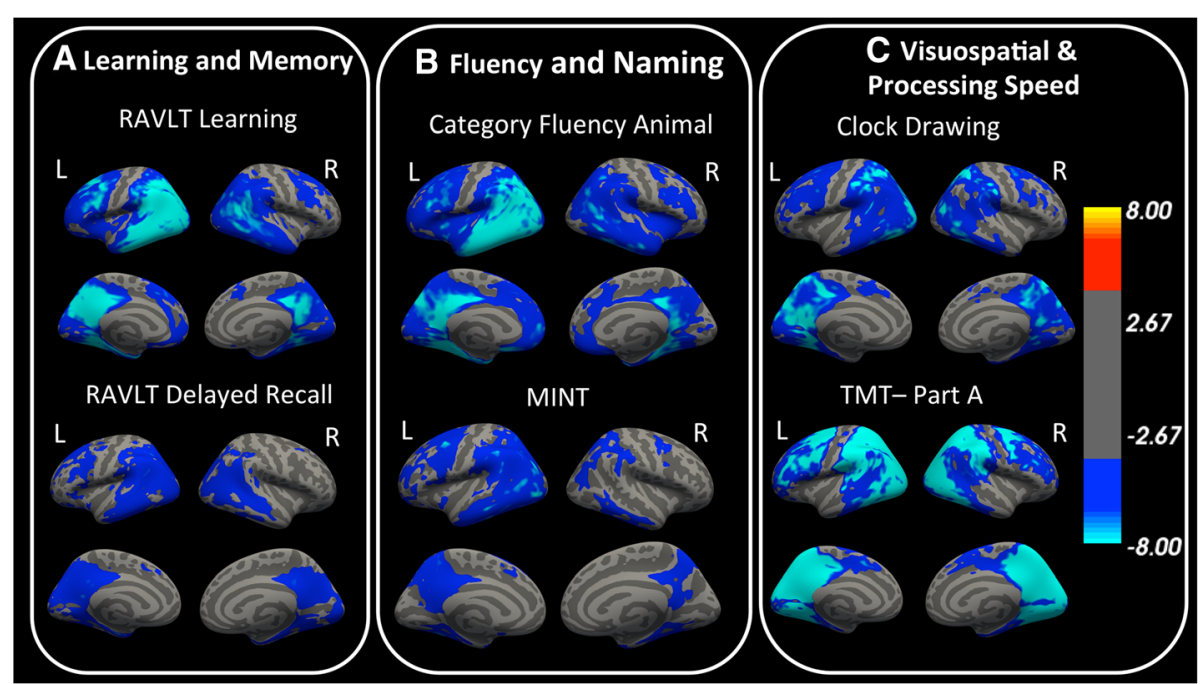

Fig. 2 Surface-based analysis demonstrates that FTP retention is associated with cognitive scores. a Performance on learning and memory tests are associated with tau in temporal, parietal, and lateral frontal cortex. b Fluency and naming abilities are related to tau in temporal, parietal, frontal, and lateral occipital cortex, with left-sided laterality. c Visuospatial function and processing speed are also related to tau in lateral frontal, lateral temporal, and parietal cortex. Plots are in - $\log (P$ value) scale and vertex values range from -log (4) to -log (8). Cool colors indicate that higher FTP retention is related to worse cognitive performance. For multiple-comparisons correction, a cluster-forming threshold of -log $(P$ value) $>4$ or $P$ value $<0.0001$, and a cluster-wise threshold $P$ value $<0.05$ were used. Results from vertex-wise analyses with $L M$, Clock Copying, and TMT part B displayed similar associations as RAVLT, Clock Drawing and TMT part A, respectively, and can be found in Additional file 1: Figure S1a. Abbreviations: RAVLT, Rey Auditory Verbal Learning Test; MINT, Multilingual Naming Test; TMT, Trail Making Test 


\section{Associations between thickness and cognitive function Learning and memory}

Worse scores on the RAVLT and LM tests were associated with thinner medial and lateral temporal cortices and with medial parietal cortices (Fig. 3a).

\section{Fluency and naming}

The topographies of the relationships between thickness and category fluency, and between thickness and MINT, were similar to the topographies from the thickness-RAVLT and thickness-LM associations (Fig. 3b). The thicknesscategory fluency association involved more medial parietal cortex than the thickness-MINT association.

\section{Visuospatial, executive function, and processing speed}

Poorer clock drawing and TMT scores were associated with reduced thickness in medial and parietal cortex, and inferior temporal cortex (Fig. 3c). Clock copying was not significantly related to thickness anywhere in the cortical mantle.

Visual assessment of the statistical maps from Figs. 1 and 2 revealed that the association between cortical thickness and cognition, are less widespread than those between FTP and cognition. Finally, in the diagnosisstratified surface-based analyses, we found no robust associations between tau and cognition or between thickness and cognition.

\section{Domain-specificity analysis}

Findings from Meng's test domain specificity analyses are shown in Fig. 4. Pairwise comparisons between taucognitive function associations revealed that the association between FTP and TMT part A was stronger in the superior parietal areas of the cortex than the other FTP-cognition associations. The relationship between FTP and RAVLT learning was stronger than that between FTP and RAVLT delayed recall in the left lateral temporoparietal cortex, left medial parietal cortex, left posterior cingulate cortex, left inferior temporal cortex, and the left dorsolateral frontal cortex. The FTPcategory fluency association was stronger in the left anterior temporal lobe, bilateral medial orbitofrontal, and bilateral insula than the FTP-TMT part A association.

The results from the domain-specificity analyses from the thickness-cognition associations are displayed in Additional file 1: Figure S2. In brief, Meng's test analyses did not elucidate any notable domain-specific correlation patterns between thickness and cognitive function.

\section{Region-of-interest analysis}

As was the case for the vertex-wise analyses, the ROI analyses showed that FTP was related to performance on each cognitive test in broad areas of the cortex (Fig. 5b). Furthermore, the ROI FTP-cognition associations showed similar topographies as the vertex-wise FTP-cognition associations. Temporal, parietal, and posterior portions of the

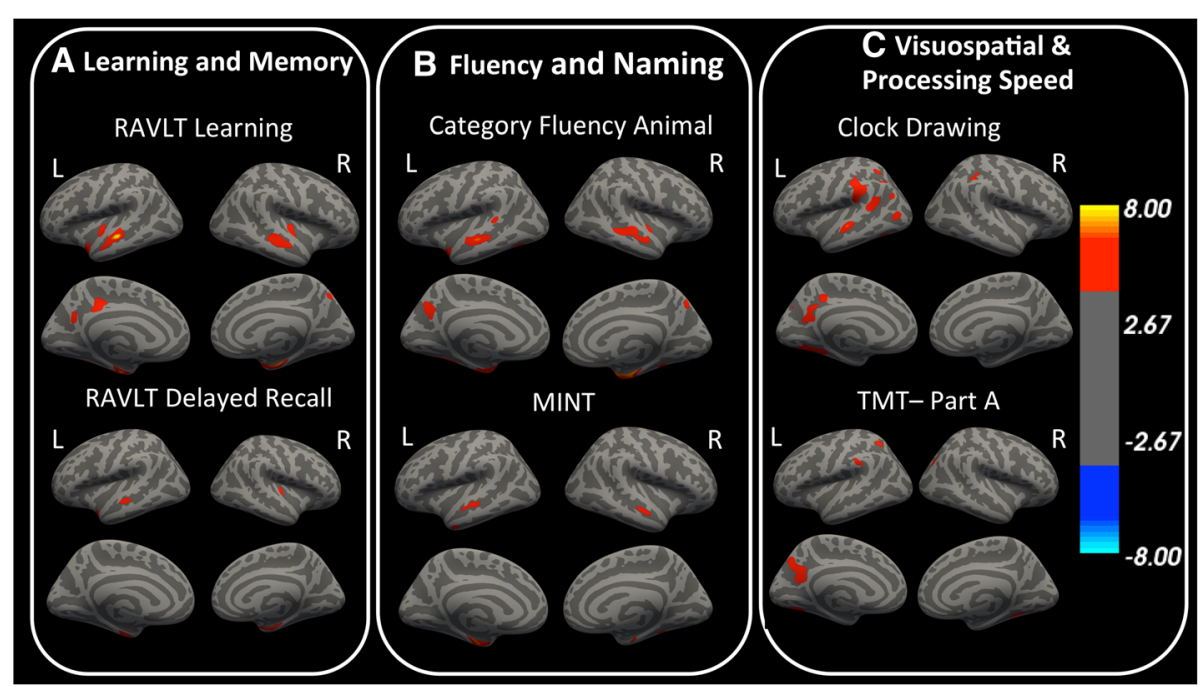

Fig. 3 Surface-based analysis demonstrates that cortical thickness is associated with cognitive scores. a Learning and memory abilities are related to cortical thickness in temporal cortex and medial parietal cortex. $\mathbf{b}$ Performance on fluency and naming tests are associated with thickness in temporal and medial parietal cortex. c Visuospatial function and processing speed are related to thickness in inferior and lateral temporal, and medial and lateral parietal cortex. Plots are in - $\log (P$ value $)$ scale and vertex values range from -log (4) to -log (8). Warm colors indicate that thicker cortex is related to better cognitive performance. For multiple-comparisons correction, a cluster-forming threshold of -log $(P$ value $)>4$ or $P$ value $<0.0001$, and a cluster-wise threshold $P$ value $<0.05$ were used. Results are shown for the same cognitive tests as Fig. 1 . Results for the rest of the cognitive tests can be found in Additional file 1: Figure S1b. Abbreviations: RAVLT, Rey Auditory Verbal Learning Test; MINT, Multilingual Naming Test; TMT, Trail Making Test 


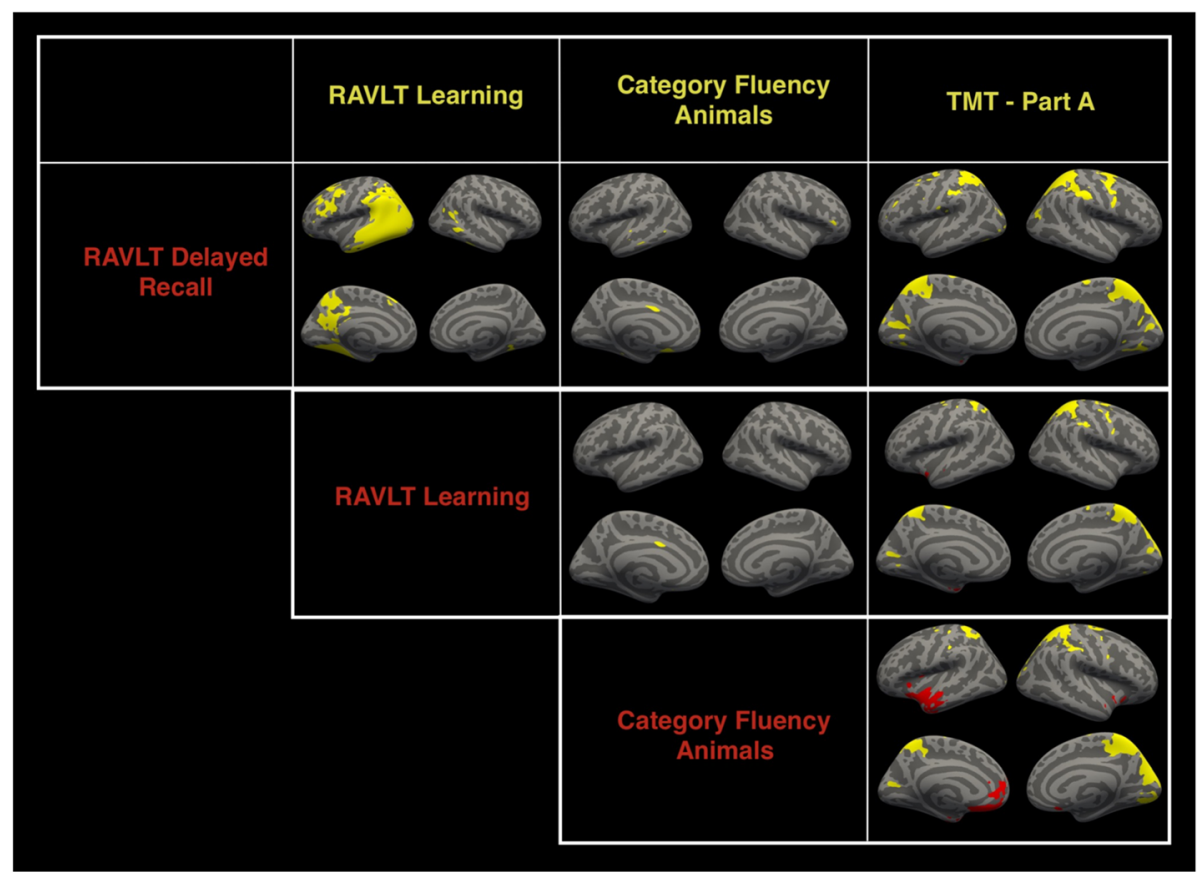

Fig. 4 Meng's test analyses show that FTP exhibits domain-specific relations with cognition. Yellow indicates that the cognitive measure noted in the column had a stronger association with FTP at that vertex than the cognitive measure noted in row ( $P$ value $<.005)$. Red indicates that the cognitive measures noted in the row had a stronger association with FTP at that vertex than the cognitive measures noted in the column $(P$ value $<0.005)$. Meng's test was limited to regions of the cortex that were significant after cluster-wise correction in the FTP-cognition or thickness-cognition analysis. Abbreviations: RAVLT, Rey Auditory Verbal Learning Test; TMT, Trail Making Test

cingulate were consistently involved across tests. However, TMT, clock drawing, and clock copying tau showed a relatively greater predilection for posterior parts of the cortex compared to tests of learning, memory, fluency, and naming tests. Regional cortical thickness was also related to cognition, though the associations were much more sparse than the ROI FTP-cognition relations (Fig. 5a). The regional thickness associations were limited to temporal cortex and left parietal cortex.

Lastly, even after accounting for regional thickness, ROI FTP remained significantly associated with cognition (Fig. 5c), though the magnitudes of the partial correlations were reduced.

\section{Discussion}

We used flortaucipir-PET and structural MRI to assess the relationships of tau and cortical thickness with performance in several domains of cognition. We found that worse cognition is related to greater tau burden and thinner cortex. We also found that the tau-cognition relationships were present in a domain-specific manner and were more widespread than the thickness-cognition relationships. We discuss our findings in the context of previous studies that have examined in vivo tau, atrophy, and cognition.
The implication of medial temporal, medial parietal, and lateral parietal tau load with memory and with global cognition has been well documented and replicated by several groups since the development of in vivo tau methods [13, 47]. Our findings are congruent with those previous studies and build upon them in a number of ways. For example, in our surface-based tau analyses, we found relatively greater left prefrontal cortex involvement in the RAVLT learning than the RAVLT delayed recall and also greater left prefrontal involvement in the LM immediate recall than LM delayed recall. This first highlights that the association between tau and AD-related learning and memory impairment is not restricted to temporal and parietal cortex. Furthermore, the implication of left prefrontal cortex is consistent with data from functional imaging and lesion studies that have localized verbal learning and verbal working memory to left frontal cortex [48, 49], suggesting that the specific location of tau deposition relates to specific aspects of cognitive loss.

The regional specificity between tau and cognitive impairment has been demonstrated in the context of early-onset, non-amnestic forms of $\mathrm{AD}$, where clinical variants exhibit different topographies of tau that are consistent with the clinical phenotype [24, 25]. There is now also mounting evidence that, even in late-onset, amnestic $\mathrm{AD}$, tau burden regionally associates with performance on 


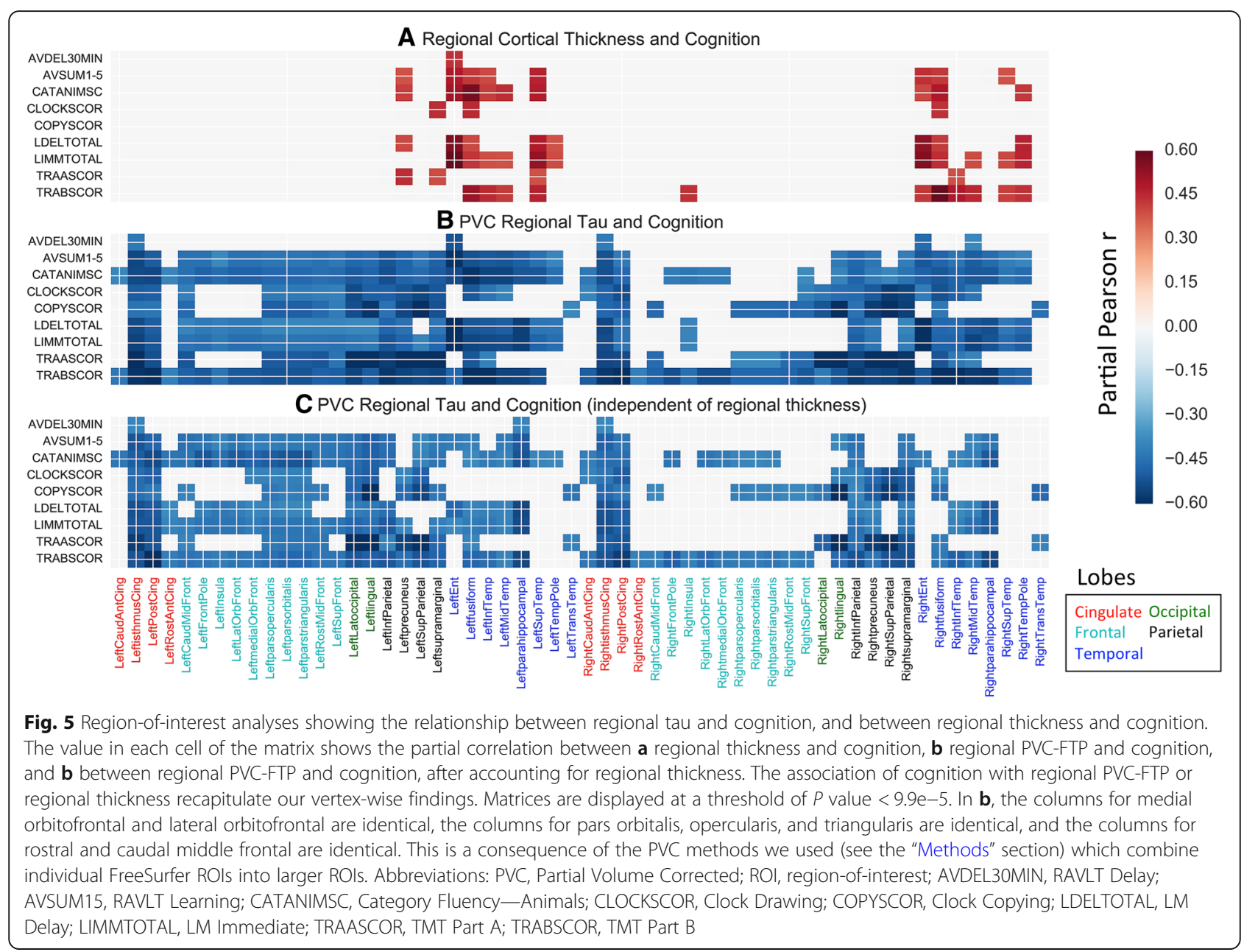

domain-specific tests of cognitive function above and beyond learning and memory [27] and that this association maps onto the cortical circuitry underlying the respective cognitive domain. This notion is supported by the significant associations between tau and tests of language, processing speed, visuospatial and executive functions, and semantic knowledge that we found in our study. Although there was spatial overlap between cognitive tests in their associations with tau, particularly in early Braak regions, there was also considerable heterogeneity. This includes left-hemispheric laterality for tests of language and stronger superior parietal involvement in tests of processing speed and constructional ability compared to tests of memory and language. These patterns of tau-cognition associations mapped closely with the cortical systems that are believed (based on functional imaging and lesion studies) to support the specific cognitive functions tested. The finding that TMT parts A and B displayed stronger associations in parietal cortex compared to tests of memory and language, for example, is congruent with fMRI studies that have shown that tasks involving numbers rely on parietal cortex [50] and that the set-shifting necessary for successful completion of TMT part B is supported by activation of intraparietal sulcus [51]. Taken together, our findings from surface-based analyses with tau-PET are aligned with recent studies of patients with early-onset [24, 25] [52] and late-onset AD [27] that have demonstrated that performance in various cognitive domains associates with tau along distinct topographic patterns.

Cortical thickness also correlated with performance across tests, and these associations were topographically similar to the tau-cognition associations. Both correlations involved temporal and parietal cortex across cognitive domains, whereas tests of processing speed and constructional abilities showed stronger correlations with tau in more posterior cortex than tests of memory and language. However, the thickness-cognition relationships were more spatially restricted than the tau-cognition relationships. Although the order in which disease-related tau and atrophy appear during aging and $\mathrm{AD}$ has been under discussion [53], models of $\mathrm{AD}$ pathophysiology have historically postulated primacy of tau pathology [54]. Our finding that the tau-cognition associations were more 
widespread than the atrophy cognition associations is consistent with this model, but future studies that assess parallel longitudinal atrophy and tau will help to clarify the temporal staging of these AD biomarkers.

Our results from the surface-based, vertex-wise analyses shown in Figs. 2 and 3 demonstrate that there are areas of the cortex where cognition is related to tau, but not to cortical thickness. This is recapitulated in our ROI analyses. We further showed in the ROI analyses that, even after taking regional thickness into account, PVC-FTP still predicted cognition. We interpret these findings to mean that tau may exert an influence on cognition via mechanisms that are independent of structural atrophy. There are several potential pathways through which tau may induce cognitive loss such as synaptic dysfunction and synaptic loss. Some of these alterations, which can now be estimated in vivo using PET [55], should be the focus of future studies to provide a better view of how the hallmark AD pathologies like tau drive decline and to realize new potential avenues for therapeutic intervention. We should note that the widespread topography of tau-cognition associations relative to thickness-cognition associations may be a consequence of deriving the tau and cortical thickness measures from different modalities. However, we applied a differential smoothing procedure to account for this and, moreover, a recent study by Bejanin and colleagues [25] also reported data suggestive of an atrophy-independent effect of tau on cognition, lending further credence to our interpretation.

Our findings have several practical implications. For example, a critical aim for designing a well-powered clinical trial is enrolling individuals that are on the verge of AD-related cognitive decline. Although our data are cross-sectional, the robust relation between tau and cognition may indicate that tau burden is a better and more sensitive predictor of imminent decline than is cortical thickness. There are several potential reasons as to why tau-PET may be a more sensitive tool for detecting decline. As we discussed above, tau may precede neurodegeneration [56] and begin to exert its affect on cognition prior to discernable atrophy. Furthermore, cross-sectional MRI-based estimates of atrophy may be affected by premorbid, inter-individual variability in thickness. Related to this, there is some evidence that atrophy measures derived from longitudinal MRI may offer predictive power beyond cross-sectional measures of atrophy in forecasting cognitive decline [57]. Thus, it will be important to incorporate longitudinal MRI if seeking to determine which imaging measures are most useful in clinical trial contexts. Lastly, because we focused on cortical patterns of tau and atrophy as they relate to cognition, we excluded hippocampal atrophy from our analyses. Hippocampal atrophy has been shown to be an indicator of imminent cognitive decline $[58,59]$ so estimates of hippocampal volume loss, in addition to cortical thinning, should be included in studies seeking to address the relative utilities of MRI and PET in predicting decline for powering clinical trials.

With further regard to clinical trials, there are now several compounds in the AD pipeline whose mechanism of action involves targeting tau pathology [60]. For these trials, it will be critical to identify individuals that are currently undergoing, or are expected to undergo, cognitive changes that are related to tau, rather than changes due to non-AD neurodegenerative, neuropsychiatric, or normal aging processes. Our data first demonstrate that noninvasive and economical neuropsychological tests like those used in ADNI can detect tau-related changes, supporting their potential utility as a screening tool. In addition, given that tau correlated with cognition in several domains, our data highlight the importance of designing neuropsychological batteries or composites for clinical trials that evaluate functions beyond memory.

Some limitations to our study should be acknowledged. FTP has been shown to exhibit off-target binding to choroid plexus in the areas adjacent to the hippocampus [61], to monoamine oxidases [62], and others. Newer generations of radiotracers that bind more specifically to tau [63] will minimize this problem in future studies. Another limitation is that our sample of participants is relatively skewed to early disease stages. This may have produced a limited range in cognitive measures and FTP signal, and hence, could have limited our ability to detect some tau-cognition and thickness-cognition relationships that may be present in AD. We were further limited by the brief battery in ADNI. Future studies with more extensive testing, for example, testing memory with nonverbal as well as verbal stimuli, will allow for broader understanding of tau-cognition relationships. The final shortcoming of our study is the small sample size, which potentially explains the failure to find meaningful tau-cognition and thickness-cognition associations in the diagnosis-stratified analyses. Future studies in larger cohorts may demonstrate relationships within diagnostic groups, helping to explain the staging of tau-atrophy relationships across the spectrum of the disease.

\section{Conclusions}

In a group of individuals along the $\mathrm{AD}$ spectrum, we found that cognitive decline in specific domains parallels the deposition of tau into the cortical systems that are thought to be responsible for subserving those domains. Our vertex and ROI analyses also revealed that there are areas of cortex where cognition is related to tau, but not cortical thickness, suggesting that tau may have an effect on cognition via mechanisms that are, at least in part, independent of significant atrophy. These data are consistent with previous studies and offer further insights into the pathophysiology underlying cognitive impairment in $\mathrm{AD}$. 


\section{Additional file}

Additional file 1: Method S1. Assessing laterality of FTP-cognition and thickness-cognition associations. Method S2. Examining the affect of $A B$ on FTP-cognition and thickness cognition associations. Figure S1. Results from vertex-wise associations between FTP and cognitive scores, and between cortical thickness and cognitive scores. Figure S2. Results from Meng's test analyses on cortical thickness-cognition correlation maps. Figure S3. Distribution of Laterality Indices (LI) from each FTP-cognition and thickness-cognition vertex-wise analysis. Figure S4. Regional PVCFTP and regional thickness are associated with cognition independent of A $\beta$ (DOCX $115582 \mathrm{~kb})$

\section{Abbreviations}

AD: Alzheimer's disease; ADNI: Alzheimer's Disease Neuroimaging Initiative; A $\beta$ : Amyloid- $\beta$; CN: Cognitively Normal; FDG: Fluorodeoxyglucose; FTP: Flortaucipir; LM: Logical Memory; MCl: Mild Cognitive Impairment; MINT: Multilingual Naming Test; MRI: Magnetic resonance imaging; PET: Positron emission tomography; PVC: Partial volume correction/corrected; RAVLT: Rey Auditory Verbal Learning Test; ROI: Region of interest; SD: Standard deviation; SE: Standard error; SUVr: Standard uptake value ratio; TMT: Trail Making Test

\section{Acknowledgements}

Data used in the preparation of this article were obtained from the Alzheimer's Disease Neuroimaging Initiative (ADNI) database (adni.loni.usc.edu). As such, the investigators within the ADNI contributed to the design and implementation of ADNI and/or provided data but did not participate in analysis or writing of this report. A complete list of ADNI investigators can be found at http://adni.loni. usc.edu/wp-content/uploads/how_to_apply/ADNI_Acknoweldgment_List.pdf

\section{Authors' contributions}

This investigation was conceptualized and designed by all authors. The data were processed and analyzed by LAD and JRM. The results from these analyses were interpreted by LAD, JRM, ETR, and SJB. The manuscript detailing our findings was drafted by LAD, ETR, and SJB. All listed authors provided critical revision of the manuscript in preparation for submission. The study was done under the supervision of SJB, JBB, and AMD. The version of the manuscript that is being submitted has been reviewed and approved by all the authors.

\section{Funding}

Data collection and sharing for this project was funded by the Alzheimer's Disease Neuroimaging Initiative (ADNI) (National Institutes of Health Grant U01 AG024904) and DOD ADNI (Department of Defense award number W81XWH-12-2-0012). ADNI is funded by the National Institute on Aging, the National Institute of Biomedical Imaging and Bioengineering, and through generous contributions from the following: AbbVie, Alzheimer's Association; Alzheimer's Drug Discovery Foundation; Araclon Biotech; BioClinica, Inc.; Biogen; Bristol-Myers Squibb Company; CereSpir, Inc.; Cogstate; Eisai Inc.; Elan Pharmaceuticals, Inc.; Eli Lilly and Company; Eurolmmun; F. Hoffmann-La Roche Ltd. and its affiliated company Genentech, Inc.; Fujirebio; GE Healthcare; IXICO Ltd.; Janssen Alzheimer Immunotherapy Research \& Development, LLC.; Johnson \& Johnson Pharmaceutical Research \& Development LLC.; Lumosity; Lundbeck; Merck \& Co., Inc.; Meso Scale Diagnostics, LLC.; NeuroRx Research; Neurotrack Technologies; Novartis Pharmaceuticals Corporation; Pfizer Inc.; Piramal Imaging; Servier; Takeda Pharmaceutical Company; and Transition Therapeutics. The Canadian Institutes of Health Research is providing funds to support ADNI clinical sites in Canada. Private sector contributions are facilitated by the Foundation for the National Institutes of Health (www.fnih.org). The grantee organization is the Northern California Institute for Research and Education, and the study is coordinated by the Alzheimer's Therapeutic Research Institute at the University of Southern California. ADNI data are disseminated by the Laboratory for Neuro Imaging at the University of Southern California.

\section{Availability of data and materials}

All neuroimaging and neuropsychology data that were used in this investigation is available online at the ADNI data repository (adni.loni.usc.edu).

\section{Ethics approval and consent to participate}

Written informed consent was provided by all study participants in accordance with the Institutional Review Boards of the respective ADNI sites.

\section{Consent for publication}

Not applicable.

\section{Competing interests}

AMD is a founder of and holds equity in CorTechs Labs, Inc. and serves on its Scientific Advisory Board. He is a member of the Scientific Advisory Board of Human Longevity, Inc. and receives funding through research agreements with General Electric Healthcare. JBB has served on advisory boards for Elan, Bristol-Myers Squibb, Avanir, Novartis, Genentech, and Eli Lilly and holds stock options in CorTechs Labs, Inc. and Human Longevity, Inc. The terms of these arrangements have been reviewed and approved by UCSD in accordance with its conflict of interest policies. LAD, JRM, ETR, and SJB declare that they have no competing interests.

\section{Author details}

${ }^{1}$ Department of Neurosciences, University of California, San Diego, 9500 Gilman Drive, La Jolla, CA 92093, USA. ²Department of Radiology, University of California, San Diego, 9500 Gilman Drive, La Jolla, CA 92093, USA. ${ }^{3}$ Center for Molecular Imaging and Genetics, University of California, 9500 Gilman Drive, San Diego, La Jolla, CA 92093, USA. Department of Psychiatry, University of California, San Diego, 9500 Gilman Drive, La Jolla, CA 92093, USA.

Received: 17 April 2019 Accepted: 8 July 2019

Published online: 27 July 2019

\section{References}

1. Weintraub S, Wicklund AH, Salmon DP. The Neuropsychological Profile of Alzheimer Disease. Cold Spring Harb Perspect Med [Internet]. 2012 [cited 2019 Jan 22];2. Available from: https://www.ncbi.nlm.nih.gov/pmc/articles/ PMC3312395/

2. Mesulam MM. Large-scale neurocognitive networks and distributed processing for attention, language, and memory. Ann Neurol. 1990;28:597-613.

3. Seeley WW, Crawford RK, Zhou J, Miller BL, Greicius MD. Neurodegenerative diseases target large-scale human brain networks. Neuron. 2009;62:42-52.

4. Donohue MC, Sperling RA, Petersen R, Sun C-K, Weiner MW, Aisen PS. Association between elevated brain amyloid and subsequent cognitive decline among cognitively normal persons. JAMA. 2017;317:2305-16.

5. Hedden T, Oh H, Younger AP, Patel TA. Meta-analysis of amyloid-cognition relations in cognitively normal older adults. Neurology. 2013;80:1341-8.

6. Jansen WJ, Ossenkoppele R, Tijms BM, Fagan AM, Hansson O, Klunk WE, et al. Association of cerebral amyloid- $\beta$ aggregation with cognitive functioning in persons without dementia. JAMA Psychiatry. 2018;75:84-95.

7. Guillozet AL, Weintraub S, Mash DC, Mesulam MM. Neurofibrillary tangles, amyloid, and memory in aging and mild cognitive impairment. Arch Neurol. 2003;60:729-36.

8. Giannakopoulos P, Herrmann FR, Bussière T, Bouras C, Kövari E, Perl DP, et al. Tangle and neuron numbers, but not amyloid load, predict cognitive status in Alzheimer's disease. Neurology. 2003;60:1495-500.

9. Nelson PT, Alafuzoff I, Bigio EH, Bouras C, Braak H, Cairns NJ, et al. Correlation of Alzheimer disease neuropathologic changes with cognitive status: a review of the literature. J Neuropathol Exp Neurol. 2012;71:362-81.

10. Xia C-F, Arteaga J, Chen G, Gangadharmath U, Gomez LF, Kasi D, et al. [18F]T807, a novel tau positron emission tomography imaging agent for Alzheimer's disease. Alzheimers Dement. 2013;9:666-76.

11. Marquié $M$, Normandin MD, Vanderburg CR, Costantino IM, Bien EA, Rycyna LG, et al. Validating novel tau positron emission tomography tracer [F-18]AV-1451 (T807) on postmortem brain tissue. Ann Neurol. 2015;78:787-800.

12. Braak H, Braak E. Neuropathological stageing of Alzheimer-related changes. Acta Neuropathol (Berl). 1991;82:239-59.

13. Schöll M, Lockhart SN, Schonhaut DR, O'Neil JP, Janabi M Ossenkoppele $\mathrm{R}$, et al. PET imaging of tau deposition in the aging human brain. Neuron. 2016:89:971-82.

14. Schwarz AJ, Yu P, Miller BB, Shcherbinin S, Dickson J, Navitsky M, et al. Regional profiles of the candidate tau PET ligand 18 F-AV-1451 recapitulate key features of Braak histopathological stages. Brain. 2016;139:1539-50. 
15. Chételat G, Desgranges B, Landeau B, Mézenge F, Poline JB, de la Sayette V, et al. Direct voxel-based comparison between grey matter hypometabolism and atrophy in Alzheimer's disease. Brain. 2008;131:60-71.

16. Dickerson BC, Bakkour A, Salat DH, Feczko E, Pacheco J, Greve DN, et al. The cortical signature of Alzheimer's disease: regionally specific cortical thinning relates to symptom severity in very mild to mild $A D$ dementia and is detectable in asymptomatic amyloid-positive individuals. Cereb Cortex N Y N 1991. 2009:19:497-510.

17. Landau SM, Harvey D, Madison CM, Koeppe RA, Reiman EM, Foster NL, et al. Associations between cognitive, functional, and FDG-PET measures of decline in AD and MCI. Neurobiol Aging. 2011;32:1207-18.

18. Joie RL, Perrotin A, Barré L, Hommet C, Mézenge F, Ibazizene M, et al. Region-specific hierarchy between atrophy, hypometabolism, and $\beta$-amyloid (Aß) load in Alzheimer's disease dementia. J Neurosci. 2012;32:16265-73

19. Harasty JA, Halliday GM, Kril JJ, Code C. Specific temporoparietal gyral atrophy reflects the pattern of language dissolution in Alzheimer's disease. Brain. 1999;122:675-86.

20. Aharon-Peretz J, Israel O, Goldsher D, Peretz A. Posterior cortical atrophy variants of Alzheimer's disease. Dement Geriatr Cogn Disord. 1999;10:483-7.

21. Lehmann M, Madison CM, Ghosh PM, Seeley WW, Mormino E, Greicius MD, et al. Intrinsic connectivity networks in healthy subjects explain clinical variability in Alzheimer's disease. Proc Natl Acad Sci. 2013;110:11606-11.

22. Lehmann M, Ghosh PM, Madison C, Laforce R, Corbetta-Rastelli C, Weiner MW, et al. Diverging patterns of amyloid deposition and hypometabolism in clinical variants of probable Alzheimer's disease. Brain. 2013;136:844-58.

23. Zhang X, Mormino EC, Sun N, Sperling RA, Sabuncu MR, Yeo BTT, et al. Bayesian model reveals latent atrophy factors with dissociable cognitive trajectories in Alzheimer's disease. Proc Natl Acad Sci. 2016;113:E6535-44.

24. Ossenkoppele R, Schonhaut DR, Schöll M, Lockhart SN, Ayakta N, Baker SL, et al. Tau PET patterns mirror clinical and neuroanatomical variability in Alzheimer's disease. Brain. 2016:139:1551-67.

25. Bejanin A, Schonhaut DR, La Joie R, Kramer JH, Baker SL, Sosa N, et al. Tau pathology and neurodegeneration contribute to cognitive impairment in Alzheimer's disease. Brain J Neurol. 2017;140:3286-300.

26. Hansson O, Grothe MJ, Strandberg TO, Ohlsson T, Hägerström D, Jögi J, et al. Tau pathology distribution in Alzheimer's disease corresponds differentially to cognition-relevant functional brain networks. Front Neurosci [Internet]. 2017 [cited 2019 Jun 11];11. Available from: https://www.ncbi.n/m.nih.gov/pmc/ articles/PMC5374886/

27. Ossenkoppele R, Smith R, Ohlsson T, Strandberg O, Mattsson N, Insel PS, et al. Associations between tau, $A \beta$, and cortical thickness with cognition in Alzheimer disease. Neurology. 2019;92:e601-12.

28. Aisen PS, Petersen RC, Donohue MC, Gamst A, Raman R, Thomas RG, et al Clinical core of the Alzheimer's disease neuroimaging initiative: progress and plans. Alzheimers Dement J Alzheimers Assoc. 2010;6:239-46.

29. Weiner MW, Veitch DP, Aisen PS, Beckett LA, Cairns NJ, Green RC, et al. The Alzheimer's Disease Neuroimaging Initiative 3: continued innovation for clinical trial improvement. Alzheimers Dement J Alzheimers Assoc. 2017;13:561-71

30. Landau SM, Marks SM, Mormino EC, Rabinovici GD, Oh H, O’Neil JP, et al. Association of lifetime cognitive engagement and low $\beta$-amyloid deposition. Arch Neurol. 2012;69:623-9.

31. Landau SM, Breault C, Joshi AD, Pontecorvo M, Mathis CA, Jagust WJ, et al. Amyloid- $\beta$ imaging with Pittsburgh compound $B$ and florbetapir: comparing radiotracers and quantification methods. J Nucl Med Off Publ Soc Nucl Med. 2013;54:70-7.

32. Jack CR, Bennett DA, Blennow K, Carrillo MC, Dunn B, Haeberlein SB, et al. NIA-AA research framework: toward a biological definition of Alzheimer's disease. Alzheimers Dement J Alzheimers Assoc. 2018;14:535-62.

33. Jovicich J, Czanner S, Greve D, Haley E, van der Kouwe A, Gollub R, et al. Reliability in multi-site structural MRI studies: effects of gradient non-linearity correction on phantom and human data. Neurolmage. 2006;30:436-43.

34. Sled JG, Zijdenbos AP, Evans AC. A nonparametric method for automatic correction of intensity nonuniformity in MRI data. IEEE Trans Med Imaging 1998;17:87-97

35. Dale AM, Fischl B, Sereno MI. Cortical surface-based analysis: I. Segmentation and surface reconstruction. Neurolmage. 1999;9:179-94.

36. Fischl B, Sereno MI, Dale AM. Cortical surface-based analysis: II: inflation, flattening, and a surface-based coordinate system. Neurolmage. 1999;9:195-207.
37. Joshi A, Koeppe RA, Fessler JA. Reducing between scanner differences in multi-center PET studies. Neurolmage. 2009;46:154-9.

38. Baker SL, Maass A, Jagust WJ. Considerations and code for partial volume correcting [18F]-AV-1451 tau PET data. Data Brief. 2017;15:648-57.

39. Fischl B, Sereno MI, Tootell RBH, Dale AM. High-resolution intersubject averaging and a coordinate system for the cortical surface. Hum Brain Mapp. 1999;8:272-84.

40. Rousset OG, Ma Y, Evans AC. Correction for partial volume effects in PET: principle and validation. J Nucl Med Off Publ Soc Nucl Med. 1998;39:904-11.

41. Desikan RS, Ségonne F, Fischl B, Quinn BT, Dickerson BC, Blacker D, et al. An automated labeling system for subdividing the human cerebral cortex on MRI scans into gyral based regions of interest. Neurolmage. 2006:31:968-80

42. Glasser MF, Smith SM, Marcus DS, Andersson J, Auerbach EJ, Behrens TEJ, et al. The human connectome project's neuroimaging approach. Nat Neurosci. 2016;19:1175-87.

43. Greve DN, Svarer C, Fisher PM, Feng L, Hansen AE, Baare W, et al. Cortical surface-based analysis reduces bias and variance in kinetic modeling of brain PET data. Neurolmage. 2014;92:225-36.

44. Hagler DJ, Saygin AP, Sereno MI. Smoothing and cluster thresholding for cortical surface-based group analysis of fMRI data. Neurolmage. 2006;33:1093-103.

45. Meng $X$, Rosenthal R, Rubin DB. Comparing correlated correlation coefficients. Psychol Bull. 1992;111:172-5.

46. Vonk JMJ, Rizvi B, Lao PJ, Budge M, Manly JJ, Mayeux R, et al. Letter and category fluency performance correlates with distinct patterns of cortical thickness in older adults. Cereb Cortex [Internet]. [cited 2019 Jan 22]; Available from: https://academic.oup.com/cercor/advance-article/doi/10.1 093/cercor/bhy138/5035452

47. Cho H, Choi JY, Hwang MS, Lee JH, Kim YJ, Lee HM, et al. Tau PET in Alzheimer disease and mild cognitive impairment. Neurology. 2016;87:375-83.

48. Nagel BJ, Herting MM, Maxwell EC, Bruno R, Fair D. Hemispheric lateralization of verbal and spatial working memory during adolescence. Brain Cogn. 2013;82:58-68.

49. Alexander MP, Stuss DT, Fansabedian N. California Verbal Learning Test: performance by patients with focal frontal and non-frontal lesions. Brain. 2003;126:1493-503.

50. Rickard TC, Romero SG, Basso G, Wharton C, Flitman S, Grafman J. The calculating brain: an fMRI study. Neuropsychologia. 2000;38:325-35.

51. Moll J, de Oliveira-Souza R, Moll FT, Bramati IE, Andreiuolo PA. The cerebral correlates of set-shifting: an fMRI study of the trail making test. Arq Neuropsiquiatr. 2002;60:900-5.

52. Phillips JS, Das SR, McMillan CT, Irwin DJ, Roll EE, Da Re F, et al. Tau PET imaging predicts cognition in atypical variants of Alzheimer's disease. Hum Brain Mapp. 2018:39:691-708.

53. Jack CR, Bennett DA, Blennow K, Carrillo MC, Feldman HH, Frisoni GB, et al. A/T/N: an unbiased descriptive classification scheme for Alzheimer disease biomarkers. Neurology. 2016;87:539-47.

54. Jack CR, Knopman DS, Jagust WJ, Shaw LM, Aisen PS, Weiner MW, et al. Hypothetical model of dynamic biomarkers of the Alzheimer's pathological cascade. Lancet Neurol. 2010;9:119-28.

55. Finnema SJ, Nabulsi NB, Eid T, Detyniecki K, Lin S-F, Chen M-K, et al. Imaging synaptic density in the living human brain. Sci Transl Med. 2016;8:348ra96

56. Jack CR, Knopman DS, Jagust WJ, Petersen RC, Weiner MW, Aisen PS, et al. Update on hypothetical model of Alzheimer's disease biomarkers. Lancet Neurol. 2013;12:207-16.

57. McEvoy LK, Holland D, Hagler DJ, Fennema-Notestine C, Brewer JB, Dale AM. Mild cognitive impairment: baseline and longitudinal structural MR imaging measures improve predictive prognosis. Radiology. 2011;259:834-43.

58. Heister D, Brewer JB, Magda S, Blennow K, McEvoy LK. Predicting MCl outcome with clinically available MRI and CSF biomarkers. Neurology. 2011;77:1619-28.

59. Spencer BE, Jennings RG, Brewer JB, Alzheimer's Disease Neuroimaging Initiative. Combined biomarker prognosis of mild cognitive impairment: an 11-year follow-up study in the Alzheimer's Disease Neuroimaging Initiative. J Alzheimers Dis JAD. 2019:68:1549-59.

60. Cummings J, Lee G, Mortsdorf T, Ritter A, Zhong K. Alzheimer's disease drug development pipeline: 2017. Alzheimers Dement Transl Res Clin Interv. 2017;3:367-84 
61. Marquié M, Verwer EE, Meltzer AC, Kim SJW, Agüero C, Gonzalez J, et al. Lessons learned about [F-18]-AV-1451 off-target binding from an autopsy-confirmed Parkinson's case. Acta Neuropathol Commun [Internet]. 2017 [cited 2019 Jan 22];5. Available from: https://www.ncbi. nlm.nih.gov/pmc/articles/PMC5648451/

62. Vermeiren C, Motte P, Viot D, Mairet-Coello G, Courade J-P, Citron M, et al. The tau positron-emission tomography tracer AV-1451 binds with similar affinities to tau fibrils and monoamine oxidases. Mov Disord Off J Mov Disord Soc. 2018;33:273-81.

63. Hostetler ED, Walji AM, Zeng Z, Miller P, Bennacef I, Salinas C, et al. Preclinical characterization of 18F-MK-6240, a promising PET tracer for in vivo quantification of human neurofibrillary tangles. J Nucl Med Off Publ Soc Nucl Med. 2016;57:1599-606.

\section{Publisher's Note}

Springer Nature remains neutral with regard to jurisdictional claims in published maps and institutional affiliations.

Ready to submit your research? Choose BMC and benefit from:

- fast, convenient online submission

- thorough peer review by experienced researchers in your field

- rapid publication on acceptance

- support for research data, including large and complex data types

- gold Open Access which fosters wider collaboration and increased citations

- maximum visibility for your research: over $100 \mathrm{M}$ website views per year

At BMC, research is always in progress.

Learn more biomedcentral.com/submissions 